\title{
OBSERVATORIO
}

\section{DISEÑO DE LA INFORMACIÓN Y PROGRESO SOCIAL: PROSPECTIVA, PERSPECTIVA Y RETOS}

\author{
Information design and social progress: Prospective, \\ perspective, and challenges
}

\section{Blas-José Subiela-Hernández}

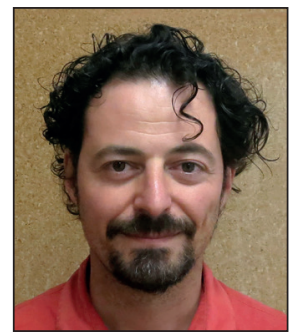

Blas-José Subiela-Hernández es doctor en comunicación por la Universidad Católica de Murcia y profesor de diseño en la Facultad de Comunicación de la misma universidad. Es investigador principal del grupo de investigación "Publicidad y RR.PP: redefiniciones, públicos y medios" y su investigación se centra en las relaciones entre el diseño publicitario y el diseño periodístico a través de la retórica visual. Es autor de varias publicaciones sobre diseño gráfico desde la perspectiva de la comunicación.

http://orcid.org/0000-0002-7540-9260

Universidad Católica San Antonio de Murcia, Facultad de Comunicación, Área de Publicidad y RRPP Campus de los Jerónimos. 30107 Guadalupe (Murcia), España bsubiela@ucam.edu

\section{Resumen}

Se define el diseño de la información en dos grandes áreas: la información periodística y la información doméstica. Y se analizan los antecedentes y las perspectivas de esta disciplina, poniendo especial atención en aquellos aspectos que pueden mejorar la sociedad. Así, desde el punto de vista del diseño periodístico, se apuesta por una mayor integración del lenguaje visual en los relatos periodísticos para aportar valor añadido a la información periodística profesional. Esta apuesta se enmarca en la necesidad de que el periodismo profesional e independiente sea valorado por la sociedad como un actor fundamental en las democracias contemporáneas. Con respecto al diseño de uso doméstico, se pone de manifiesto la necesidad de rediseñar, con criterios de claridad, responsabilidad y transparencia, documentos como contratos, facturas o etiquetado de productos, para que prime en ellos el derecho a una comunicación clara, por encima de otros intereses comerciales.

\section{Palabras clave}

Diseño de la información; Diseño gráfico; Arquitectura de la información; Visualización de la información; Información periodística; Información de uso doméstico; Periodismo visual; Comunicación clara; Diseño responsable, Derecho a la comunicación.

\begin{abstract}
Information design is defined in two areas: journalistic information and domestic information. In this study the background and perspective of Information design are analyzed -paying special attention to those aspects that can improve society. Also, from the viewpoint of journalistic design, we focus on greater integration of visual language in news stories as a tool to add value to professional journalistic information. This commitment is framed by the need for professional and independent journalism to be valued by society as a fundamental actor in contemporary democracies.In domestic information design, we highlight the need to redesign, with criteria for clarity, responsibility, and transparency in documents such as contracts, invoices, and product labeling, giving priority to clear communication above commercial interests.
\end{abstract}

\section{Keywords}

Information design; Graphic design; Information architecture; Information visualization; Journalistic information; Domestic information; Visual journalism; Clear communication; Responsible design; Right to communication.

Subiela-Hernández, Blas-José (2017). "Diseño de la información y progreso social: prospectiva, perspectiva y retos". El profesional de la información, v. 26, n. 6, pp. 1019-1024. 


\section{Introducción: definición y delimitación}

El origen del concepto de diseño de la información está ligado a otros como arquitectura de la información (Wurman, 1997) o visualización de la información (Card; Mackinlay; Shneiderman, 2000). E incluso se identifica en ocasiones con la infografía (Vargas-Jiménez; Greiff-Tovar; Rojas-Ramírez, 2014) y con lo que autores como Costa (1998) han denominado esquemática.

Una de las definiciones pioneras sobre diseño de la información (Horn, 2000, p. 15) lo describe como

"arte y ciencia de preparar la información para que pueda ser utilizada por los seres humanos de una forma eficiente y efectiva"1.

Definiciones posteriores como la de la Society for Technical Communication (Baer; Vacarra, 2008), lo identifican como la transformación de datos complejos en mensajes comprensibles para los usuarios ${ }^{2}$.

De modo que la evolución del concepto ha pasado a considerar como objeto del diseño de la información a los datos, que pueden ser considerados como un tipo muy concreto de información. Precisamente en la delimitación del término "información" reside la clave para la definición concreta de esta disciplina. No obstante, antes de profundizar en el objeto del diseño de la información, consideramos que es una disciplina que deriva del diseño gráfico: podemos afirmar que el diseño de la información no es más que el diseño gráfico aplicado a la información.

Cuando aquí hablamos de información nos referimos fundamentalmente a información periodística. $Y$ a ella dedicaremos una parte importante de este texto. Pero también debemos hacer referencia a otro tipo de información. Frascara (2000, p. 103) considera que el diseño gráfico para la información incluye el diseño editorial (libros, revistas, diarios...), el de tablas alfanuméricas, gráficos y diagramas, manuales de instrucciones (uso de aparatos, reglas de juegos...), señalización (símbolos, carteles, señales y sistemas) y mapas y planos (sin el detalle de la cartografía, la arquitectura o la ingeniería).

Sin ser tan preciso como Frascara, pero en un sentido muy similar, Horn (2000, p. 15) afirma que el objeto del diseño de información es el desarrollo de documentos que sean comprensibles, rápidos, precisos y fáciles de convertir en una acción efectiva ${ }^{3}$.

Por lo tanto, el diseño de la información no sólo abarca a la información periodística, sino que también hace referencia a la que denominaremos, sin afán taxonómico, "información de uso doméstico".

Se trata de una información que está imbricada en nuestra vida y que pasa habitualmente desapercibida. Más allá de los clarificadores ejemplos propuestos por Frascara, podemos citar también otros especialmente interesantes desde nuestro punto de vista: facturas de compañías de suministro de servicios, etiquetas de productos y envases, contratos... Información que, en la mayoría de las ocasiones, procede de organizaciones comerciales, pero que no tiene objetivos publicitarios. No tendremos en cuenta dentro del diseño de la información otros ámbitos del diseño comercial con fines publicitarios y promocionales, ya que entendemos que en estos casos los objetivos de la comunicación van mucho más allá de la información.

Para la definitiva acotación del concepto de diseño de la información es necesario tener presente que la información es inseparable del diseño. No se puede transmitir información sin darle una forma determinada. Porque la información es un producto intangible, que se vuelve tangible cuando se plasma en un soporte por medio de un diseño. El papel del diseño en la construcción de la información es por tanto imprescindible. Del mismo modo que es imprescindible la gramática para el correcto manejo de un idioma, o es imprescindible encuadrar la escena para realizar una fotografía.

Esta observación es especialmente pertinente porque con demasiada frecuencia el diseño se considera simplemente un accesorio de la información, un "adorno" prescindible, un extra que no puede ni aumentar ni reducir el valor de los mensajes a los que paradójicamente da forma. Y sin embargo es en gran medida el responsable de que la información despierte el interés y llegue en condiciones óptimas a sus audiencias. Por ello es imprescindible, también desde el ámbito académico, reivindicar la importancia del diseño de la información como una variable decisiva para aportar valor a la comunicación.

El diseño es en gran medida el responsable de que la información despierte el interés y llegue en condiciones óptimas a sus audiencias

\section{Diseño de información periodística. Origen, evolución y perspectivas}

Aunque la vinculación de periodismo y diseño es evidente, las posibilidades del último han estado siempre condicionadas por los avances de la técnica. Por eso hasta el descubrimiento de la fotografía y la aplicación de sus principios químicos al mundo de las artes gráficas, las posibilidades del diseño gráfico en la información eran limitadas. Y no fue hasta la segunda mitad del siglo XX que el diseño gráfico empezó a ser considerado como una disciplina que requería la atención de informadores y medios de comunicación en general. Han sido protagonistas imprescindibles en este proceso profesionales como Mario García, Edmund C. Arnold o Daryl Moen, pioneros en la integración del diseño periodístico como un elemento más del proceso de comunicación establecido entre lector y periódico. En España marcó un hito el lanzamiento del diario El país y a lo largo de las dos últimas décadas del siglo XX se publicaron numerosos manuales sobre diseño de la información (Canga-Larequi, 1994; García-Yruela, 2003; González-Díez; Pérez-Cuadrado, 2001; Zorrilla-Ruiz, 2002), especialmente centrados en la diagramación de los diarios. Desde finales del siglo pasado el papel del diseño en los diarios quedó perfectamente definido, tanto desde el punto de vista de la práctica profesional como desde el académico. 
La irrupción de internet y la adaptación de los medios al universo multimedia supuso un nuevo reto para el diseño, que vio de nuevo condicionadas sus posibilidades por factores tecnológicos (Armentia-Vizuete, 2005). Hay intentos por aplicar los mismos principios desarrollados para el papel en el nuevo soporte, como por ejemplo la creación de pdfs interactivos y enriquecidos con contenidos multimedia, pero basados en estructuras modulares. Sin embargo, hasta que internet no alcanza cierta madurez como medio y se estudian los hábitos de consumo de información de los internautas, el diseño gráfico aplicado a la información en las pantallas es errático.

\section{En el diseño periodístico contemporá- neo es necesario diferenciar el estilo vi- sual de la información diaria (noticias de actualidad) del de textos menos urgen- tes (reportajes y entrevistas)}

En la actualidad cada soporte (papel y pantallas) ha desarrollado su propio lenguaje visual, de acuerdo a sus posibilidades tecnológicas y sobre todo a las características de consumo de sus usuarios. El resultado es un tanto sorprendente, porque el diseño periodístico en internet ha generado estructuras lineales en las que las posibilidades narrativas del grafismo son limitadas. Frente a una puesta en página cada vez más cuidada de la prensa escrita, se impone el diseño plano y más abierto de las pantallas, en las que el relato informativo puede complementarse con diversas fuentes y recursos a golpe de clic. La necesidad del medio impreso de ofrecer toda la información en el espacio limitado de la página hace necesario que el diseño presente todos los recursos de la noticia de forma organizada y plana, frente a la profundidad posible en el medio electrónico.

Sea en pantalla o en soporte impreso, la relevancia del diseño periodístico en la actualidad es indiscutible. Convocatorias como los premios Malofiej de infografía o los ÑH sobre diseño periodístico, ambas avaladas por la Society for News Design en su capítulo español, son una muestra más de ello.

Disciplinas como la infografía han pasado de ser meros complementos de la información a definirse cada vez más como un nuevo género periodístico autosuficiente (Abreu-Sojo, 2002) para explicar acontecimientos complejos de forma eficaz y atractiva. A partir del desarrollo de la infografía surgen modelos nuevos bajo la denominación de visualización de la información (o visualización de datos), que permiten también dar un acceso visual y sencillo a las cada vez más ingentes cantidades de datos con los que se enfrentan las sociedades contemporáneas.

Esta modalidad ha sido ya tratada en un monográfico de EPI (EPI SL, 2014), por lo que no nos detendremos aquí tanto en esta parte del diseño de la información y sí mucho más en lo que tiene que ver con la puesta en página de todos los textos que configuran la información periodística. Entiéndase que adoptamos aquí una perspectiva semiótica con respecto a la idea del texto, de modo que tomamos como texto el relato periodístico completo, con sus diferentes niveles verbales (titulares, entradillas, sumarios, etc.) y visuales (gráficos, fotos, retículas, formas, colores, etc.).

Dentro del diseño periodístico contemporáneo es necesario diferenciar el estilo visual de la información diaria (noticias de actualidad), del de textos menos urgentes (reportajes y entrevistas). En el diseño de la información diaria de actualidad (tanto en soporte impreso como digital) las alternativas compositivas están determinadas por la modulación del espacio (que suele ser estático) y por el principio de jerarquización. Además, las rutinas productivas de la prensa diaria, su inmediatez y la necesidad de optimizar los recursos humanos y materiales en la permanente crisis de los medios condicionan también un diseño estructural y previsible (Subiela-Hernández; Gobantes-Bilbao, 2009). En estos casos el impacto visual debe proceder de las imágenes (fotografías, ilustraciones, infografías, gráficos...), mientras que la puesta en página se reduce a la repetición de prototipos y al ajuste de los textos. Por otra parte la búsqueda de la máxima objetividad en el tratamiento de esta información aleja también una integración mayor del diseño en el relato. Se trata de un diseño que aspira a la denominada retórica de la neutralidad (Kinross, 1989), aunque como el propio autor pone de manifiesto, dicha neutralidad resulte imposible de alcanzar.

Sin embargo a medida que los contenidos permiten un enfoque más profundo y más alejado de la inmediatez, el diseño puede tener una mayor importancia para construir el relato periodístico. Cabe destacar el trabajo de Rodrigo Sánchez en el diario El mundo, especialmente en las portadas de su suplemento cultural La Luna de metrópoli o en algunos de los cuadernillos monográficos incluidos en el periódico. Como el propio periodista y diseñador explica (Ryan; Conover, 2004, p. 134) "Great design synthesizes content" (un gran diseño sintetiza el contenido), afirmación que conden-

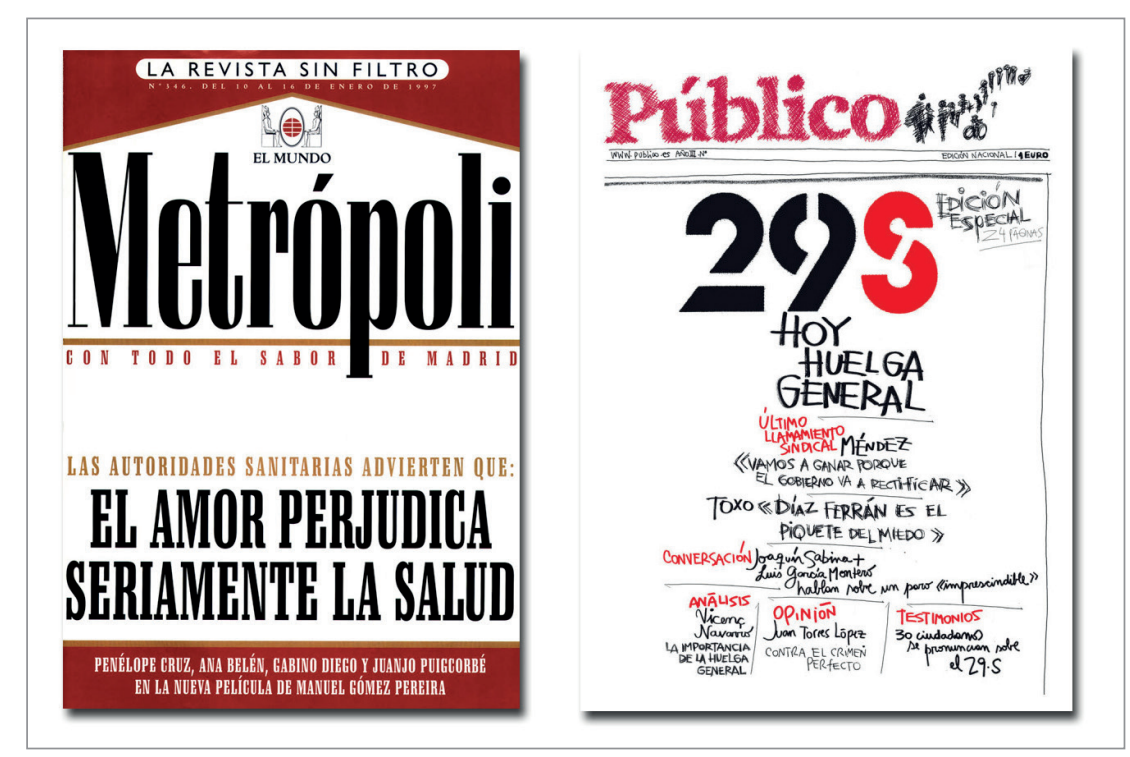

Figura 1. Dos ejemplos en los que el diseño aporta información al relato periodístico 
sa muy bien los fundamentos de un diseño que forma parte de la información y no se limita a hacerla legible. En esta línea, es también destacable el trabajo de Diego Areso al frente del equipo de diseño del diario Público en su versión impresa, ya desaparecida. Este periódico fue probablemente el mejor ejemplo de cómo se puede conseguir la máxima integración entre el relato lingüístico y el relato visual, especialmente en sus portadas. Hablamos aquí de un diseño capaz de formar parte del discurso informativo, aportando sentido y ayudando a una mejor comprensión del mensaje. En palabras de Tena-Parera (2004, p. 2), el diseño

"permite aportar al mensaje global elementos informativos que van más allá de la información meramente textual".

La diferencia planteada entre el diseño de las páginas de información de actualidad diaria y aquellas otras dedicadas a temas menos urgentes parece definir el futuro más inmediato de la disciplina y de los propios medios informativos.

Aunque es un debate en el que no vamos a entrar aquí, la misión de los diarios impresos ya no es la de proporcionar las últimas noticias de forma urgente; el resto de medios cumple esta función de una forma más eficaz desde hace décadas. Y las redes sociales, con los usuarios convertidos en informadores (que no periodistas), compiten con los medios electrónicos en la inmediatez de la información (que no en su calidad).

\section{A medida que la información se vuelve más compleja, el diseño toma más im- portancia no sólo como contenedor de la noticia, sino como contenido informa- tivo por sí mismo}

Por eso cada vez son más frecuentes $-\mathrm{y}$ recomendableslos monográficos en los que se afronta con profundidad y reflexión otro tipo de información más compleja y que demanda, entre otras cosas, un diseño más vinculado al relato, aunque para ello deje de respetar estructuras y retículas. EI diseño estructural, lineal, reticular, queda reservado para la noticia de actualidad. Tanto en las páginas impresas, en las que este tipo de información tiene cada vez menos presencia, como en las electrónicas. Porque es este tipo de diseño "mecánico", de estructuras repetidas, el que también utilizan los medios digitales para presentar la actualidad más inmediata, tanto en sus ediciones electrónicas como en sus redes sociales. Además, como ya se ha puesto de manifiesto, es un tipo de diseño funcional, que optimiza la legibilidad y garantiza un acceso rápido a la información.

A medida que la información se vuelve más compleja y requiere un mayor esfuerzo del periodista por hacerla accesible, el diseño va tomando importancia no sólo como contenedor de la noticia, sino como contenido informativo por sí mismo. Y esto nos debe llevar a la siguiente reflexión: el diseño periodístico debe ser utilizado como un recurso fundamental en el proceso de dotar de valor añadido a la información periodística. El futuro del periodismo debe pasar por la elaboración de información de calidad por la que el lector esté dispuesto a pagar (Errea-Múgica, 2015). Y en esa elaboración, el diseño no puede quedar al margen.

La academia, mientras tanto, debería ser capaz de realizar más allá del análisis descriptivo, investigaciones que permitieran conocer mejor a los lectores y a sus motivaciones, de modo que se generen propuestas, desde el grafismo, que sirvan para aumentar el valor percibido de la información periodística de calidad. Sólo así se podrá contribuir al mantenimiento del periodismo independiente y riguroso, imprescindible -más que nunca- para el mantenimiento de las democracias contemporáneas.

El diseño es en gran medida el responsable de que la información despierte el interés y llegue en condiciones óptimas a sus audiencias

\section{Información de uso doméstico y derecho a la comunicación clara}

El diseño gráfico también debe cumplir un papel fundamental en lo que denominamos información de servicio o doméstica. Dos ejemplos servirán para definir claramente este ámbito del diseño de la información: un horario de trenes es un documento informativo de servicio en el que el diseño puede hacer que el contenido sea comprendido y asimilado rápidamente o que, por el contrario, la información no llegue con claridad a los usuarios. Otro ejemplo son los contratos o las facturas de suministro eléctrico o de telefonía. Ambos tipos de documentos pueden ser ininteligibles o accesibles en función del diseño.

En el caso de la información periodística, es evidente que el diseño siempre perseguirá transmitir la noticia de la forma más clara y precisa posible. Sin embargo, la comunicación comercial y los documentos que genera (entre los que se encuentran los que hemos denominado de uso doméstico) son denunciados en demasiadas ocasiones por su falta de claridad.

Por ejemplo, la propia Comisión Nacional del Mercado y la Competencia (CNMC) ha detectado que el $46,5 \%$ de los españoles no entienden su factura de la luz (CNMC, 2016). En este bajo nivel de comprensión sería interesante determinar la influencia que tiene la composición visual de las mismas -composición que la mayoría de compañías toman de la plantilla propuesta por el Estado a través del Boletín oficial del Estado (España, 2014) (figura 2).

También la banca ha sido protagonista de situaciones en las que una información poco clara sobre determinados productos financieros (véase el caso de las acciones preferentes emitidas por Bankia) ha sido determinante para definir la responsabilidad de los clientes ante los tribunales. De hecho, entidades como BBVA han integrado el principio de comunicación clara, transparente y responsable dentro de sus políticas de responsabilidad social corporativa desde 2015 (Rubio, 2015), considerando bajo este principio no sólo cuestiones lingüísticas, sino también de diseño de la información. 


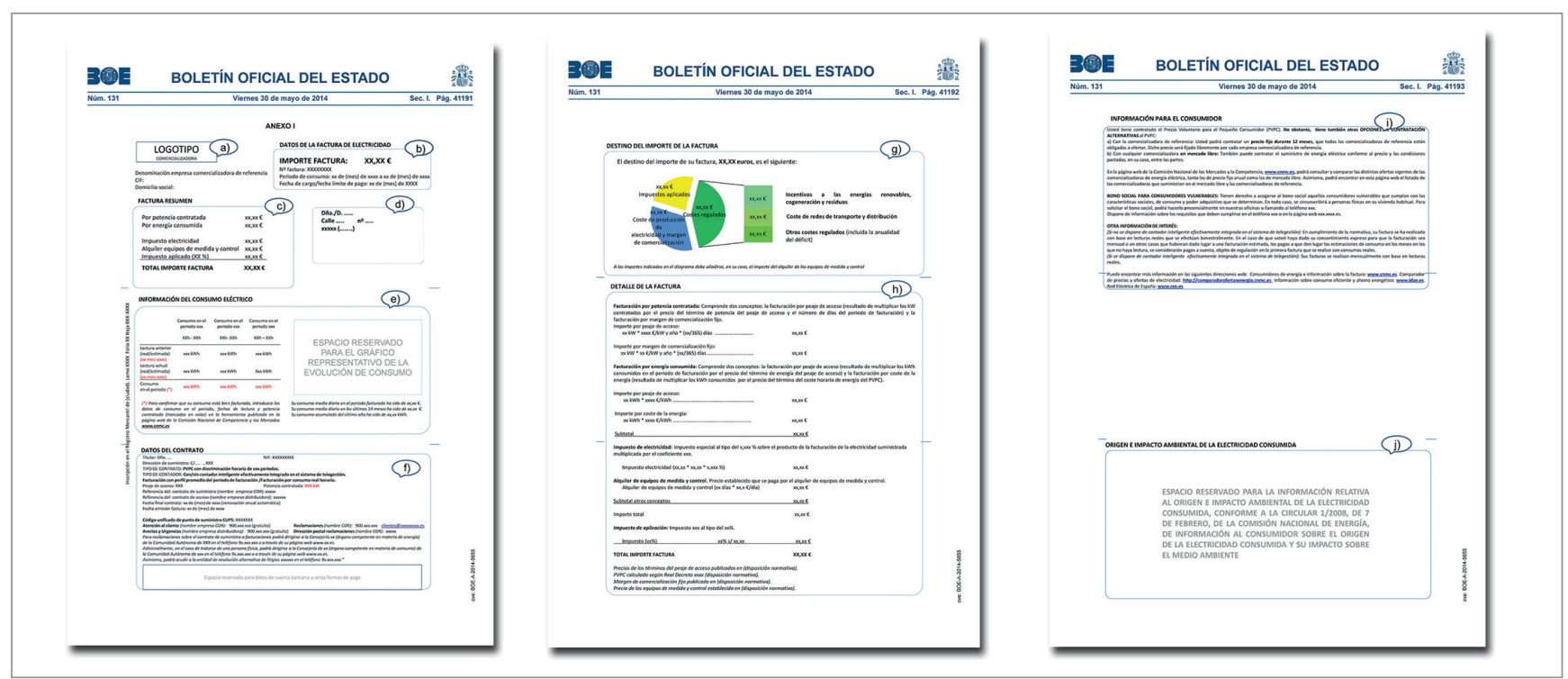

Figura 2. Plantilla de diseño publicada en el $B O E$ para las facturas de suministro de electricidad

La comunicación clara, en la que el diseño de la información debe tener un papel fundamental, se está configurando como un derecho de los ciudadanos y una obligación de las organizaciones en las sociedades avanzadas (Montolío; Tascón, 2017), más allá de las obligaciones derivadas para las administraciones públicas de la Ley de Transparencia (España, 2013). Por ello creemos firmemente que el futuro del diseño de la información de uso doméstico tendrá que desarrollarse forzosamente bajo las premisas de la claridad. Premisas que, desde un punto de vista teórico, están plasmadas en un conjunto de principios tipográficos y compositivos que garantizan como mínimo la legibilidad del documento diseñado (Unger, 2009). Y que no son respetadas por numerosos documentos de uso doméstico.

Un ejemplo claro son las casi infinitas y tipográficamente diminutas listas de cláusulas presentes en los reversos de la mayoría de contratos comerciales. La composición tipográfica de estos textos suele caracterizarse por el empleo de cuerpos de texto por debajo de los 7 puntos, líneas con una longitud excesiva e interlineado insuficiente. Se trata de características identificadas en cualquier manual de diseño como contrarias a la legibilidad. Y, sin embargo, se utilizan. Lo que forzosamente nos lleva a pensar que el objetivo perseguido al utilizarlas es precisamente dificultar la legibilidad.

Capítulo aparte merecería también el diseño de las etiquetas y los envases de los productos de consumo, especialmente en el sector de la alimentación. Aunque la legislación obliga a incluir información nutricional en todos los productos de alimentación, ésta suele quedar relegada a los lugares menos visibles mientras que en el espacio principal del soporte el diseño ofrece composiciones que, con frecuencia, llevan al engaño. Este tipo de diseños han sido denunciados por algunos consumidores con movimientos en redes sociales como el abanderado bajo el hashtag \#etiquetareal

Es necesario que desde la comunidad científica se ponga en evidencia, primero, que el diseño debe ayudar al usuario. $Y$ segundo, que en muchos casos no lo está haciendo. Sería beneficioso promover investigaciones que permitan identificar diseños contrarios a la claridad y que a la vez pro- pongan soluciones para que dichos diseños se alejen de la confusión y de la desinformación y se pueda establecer un equilibrio entre los intereses de la sociedad y los intereses comerciales. El diseño al servicio de la comunicación clara debe integrarse en las políticas de responsabilidad social de las organizaciones comerciales. Y debe ser considerado un activo para la construcción de su buena reputación.

\section{A modo de conclusión}

El diseño de la información es una disciplina tan amplia como su propio objeto de estudio porque toda información debe tener un diseño para ser transmitida. Sólo por esta razón ya tienen una importante responsabilidad social aquellos profesionales que lo ejercen. Y también aquellos que se dedican a investigarlo. Hemos centrado nuestra atención en dos tipos de información para poder demostrar esta afirmación de un modo más preciso:

- En el caso de la información periodística, el diseño debe ser considerado como un elemento esencial para añadir valor al periodismo profesional e independiente. La sociedad debe ser consciente de que merece la pena pagar por la información periodística elaborada con estándares profesionales y con independencia. Y una forma de diferenciar esta información, más allá del propio relato verbal y sus estándares de calidad, debe pasar por una mejor integración del diseño, del relato visual, dentro del complejo relato periodístico. A los investigadores corresponde estudiar los hábitos de consumo de los lectores, sus motivaciones, sus intereses, etc. para proporcionar soluciones que garanticen la viabilidad del periodismo independiente.

- Con respecto a lo que hemos llamado información de uso doméstico, es necesario promover el derecho a una comunicación clara, transparente y responsable, para que no se vuelvan a repetir situaciones tan graves como las provocadas por la información confusa de algunos productos financieros en la reciente crisis económica. También aquí el diseño de la información tiene una responsabilidad importante, porque factores como la legibilidad o la jerarquización son deficientes en muchos de los documentos de uso doméstico de los que hemos hablado a lo largo 
de este texto. Las organizaciones deben comprender que adoptar estrategias de comunicación clara con sus clientes redundará en una mejor percepción de las mismas y contribuirá a la mejora de su reputación.

En este caso la academia debe colaborar con las asociaciones de consumidores en identificar documentos de uso doméstico con diseños deficientes, susceptibles de confundir y desinformar y debe proponer soluciones para dichos diseños a partir de la investigación sobre la percepción, recepción y comprensión de los mismos.

\section{Notas}

1. Traducción propia del texto original en inglés:

"Information design is defined as the art and science of preparing information so that it can be used by human beings with efficiency and effectiveness".

2. Traducción propia del texto original en inglés:

"The translating of complex, unorganized, or unstructured data into valuable, meaningful information".

3. Traducción propia del texto original en inglés:

"To develop documents that are comprehensible, rapidly and accurately retrievable, and easy to translate into effective action".

\section{Referencias}

Abreu-Sojo, Carlos (2002). “¿Es la infografía un género periodístico?". Revista latina de comunicación social, n. 51. https://www.ull.es/publicaciones/latina/2002abreujunio5101. htm

Armentia-Vizuete, José-Ignacio (2005). "Los diarios digitales siguen buscando su propia identidad tras una década de existencia". Estudios sobre el mensaje periodístico, n. 11, pp. 9-22. https://goo.gl/TWPWH7

Baer, Kim; Vacarra, Jill (2008). Information design workbook. Beverly, Mass: Rockport. ISBN: 9781592534104

Canga-Larequi, Jesús (1994). El diseño periodístico en prensa diaria. Barcelona: Bosch. ISBN: 8476762941

Card, Stuart K.; Mackinlay, Jock D.; Shneiderman, Ben (2000). Readings in information visualization: Using vision to think. San Francisco: Morgan Kaufmann. ISBN: 9781 558605336

CNMC (2016). "Casi la mitad de los usuarios no entiende su factura de la luz". Comisión Nacional de los Mercados y la Competencia, 13 mayo.

https://goo.gl/CvyAo5

Costa, Joan (1998). La esquemática: visualizar la información. Barcelona: Paidós ISBN: 9788449306112

EPI SL (2014). "Visualización de la información". El profesional de la información, v. 23, mayo-junio, n. 3, pp. 213-338. http://www.elprofesionaldelainformacion.com/contenidos/2014/ mayo/index.html

Errea-Múgica, Javier (2015). El diario o la vida. Una defensa a tiros de los periódicos y del periodismo. Pamplona: Libros del Cuerno. ISBN: 9788460840114
España (2013). "Ley 19/2013, de 9 de diciembre, de transparencia, acceso a la información pública y buen gobierno". $B O E$, n. 295, 10 de diciembre. https://goo.gl/a5B2xJ

España (2014). "Resolución 5655 de 23 de mayo, de la Dirección General de Política Energética y Minas, por la que se establece el contenido mínimo y el modelo de factura de electricidad". BOE, n. 131, 30 de mayo. https://goo.gl/tt3yW7

Frascara, Jorge (2000). Diseño gráfico para la gente. Buenos Aires: Infinito. ISBN: 9789879637050

García-Yruela, Jesús (2003). Tecnología de la comunicación e información escrita. Madrid: Síntesis. ISBN: 98784 99582740

González-Díez, Laura; Pérez-Cuadrado, Pedro (2001). Principios básicos sobre diseño periodístico, Madrid: Universitas. ISBN: 9878479911157

Horn, Robert E. (2000). "Information design: Emergence of a new profession". En: Jacobson, Robert (ed.). Information design. Cambridge: MIT Press. ISBN: 9780262100694 https://goo.gl/Li7dEr

Kinross, Robert (1989). "The rhetoric of neutrality". En: Margolin, Victor (ed.). Design discourse: History, theory, criticism. Chicago: University Chicago Press, pp. 131-143. ISBN: 9780226505145 http://public.wsu.edu/ arola/356/spring09/kinross.pdf

Montolío, Estrella; Tascón, Mario (2017). “Comunicación clara". Prodigioso volcán. https://goo.gl/x7Jihd

Rubio, Loreto (2015). Os necesito a todos. Madrid: LID Editorial. ISBN: 9788483564783

Ryan, William; Conover, Theodore (2004). Graphic communications today. New York: Thomson/Delmar Learning. ISBN: 9780766820753

Subiela-Hernández, Blas-José; Gobantes-Bilbao, Maite (2010). "La retórica visual en el diseño periodístico: un diagnóstico". En: II Jornadas de diseño en la sociedad de la información, pp. 31-39. ISBN: 9788469533895

https://dialnet.unirioja.es/descarga/articulo/4165956.pdf

Tena-Parera, Daniel (2004). Diseño gráfico y comunicación. Madrid: Pearson Educación. ISBN: 9788420542300

Unger, Gerard (2009). ¿Qué ocurre mientras lees? Tipografía y legibilidad. Valencia: Campgràfic. ISBN: 9788496657120

Vargas-Jiménez, Sandra-Paola; Greiff-Tovar, Boris-Alexander; Rojas-Ramírez, Óscar (2014). “¿Infografía... visualización... diseño de información? En busca de los indicios de su configuración y delimitación como campo disciplinar". Kepes, n. 10, pp. 105-141.

http://vip.ucaldas.edu.co/kepes/downloads/Revista10_7.pdf

Wurman, Richard S. (1997). Information architects. Michigan: Graphis. ISBN: 9781888001389

Zorrilla-Ruiz, Jesús (2002). Introducción al diseño periodístico. Pamplona: Eunsa. ISBN: 9788431320324 